\title{
Disseminated Coccidioidomycosis of the Rearfoot: A case report
}

\author{
by Robert Frykberg, DPM, MPH ${ }^{1} \rrbracket$, Beth Noe, DPM ${ }^{2}$, Stephanie Michael, DPM ${ }^{3}$, Edward \\ Tierney, DPM ${ }^{4}$
}

The Foot \& Ankle Journal 1 (8): 1

Coccidioides immitis is a fungus endemic to the Southwestern United States, Mexico, and parts of Central and South America. While some populations are more at risk for the infection, most persons infected with the fungus remain asymptomatic. However, up to one percent of these individuals will go on to develop the disseminated form of the disease. Manifestations of this secondary type of infection may involve both soft tissue and bone. We present a case report of a patient presenting with a painful ankle who was found to have diffuse coccidioidal osteomyelitis. After a confirmatory biopsy and culture, long term fluconazole therapy was initiated as the recommended treatment. Although the disseminated form of the disease is relatively uncommon, it is important to include the infection as a differential diagnosis in patients living in endemic areas who present with suspicious bone lesions.

Key words: Coccidiodes immitis, Coccidioidomycosis

Accepted: July $2008 \quad$ Published: August 2008

This is an Open Access article distributed under the terms of the Creative Commons Attribution License. It permits unrestricted use, distribution, and reproduction in any medium, provided the original work is properly cited. @The Foot \& Ankle Journal (www.faoj.org)

Disseminated Coccidioides immitis is rarely a causative organism in osteomyelitis. However, in certain parts of the Western Hemisphere, coccidioidomycosis (Valley Fever) is endemic and should be considered as part of the list of differential diagnoses in osteomyelitis. The infection is most prevalent in the desert region of the southwestern United States which includes parts of New Mexico, Arizona, California, Nevada, and Texas. ${ }^{1,2,3}$

Address correspondence to: Robert Frykberg, DPM, MPH

Carl T. Hayden VA Medical Center, 650 E. Indian School Rd.,Phoenix, AZ

85012. email: Robert.Frykberg@med.va.gov

${ }^{1}$ Chief of Podiatry, Carl T. Hayden VA Medical Center, 650 E. Indian School Rd. , Phoenix, AZ 85012.

${ }^{2}$ Beth Noe, DPM , Attending Podiatrist, Carl T. Hayden VA Medical

Center.

${ }^{3}$ Stephanie Michael, DPM Private Practice, Atlanta, GA. Former $3^{\text {rd }}$

year resident Carl T. Hayden VA Medical Center.

${ }^{4}$ Edward Tierney, DPM Attending Podiatrist, Carl T. Hayden VA

Medical Center.
There are an estimated 1,000,000 coccidioidal infections per year in the United States. ${ }^{1}$ Earthquakes, dust storms, archaeological digs, construction work, and military training exercises have been associated with infection due to Coccidioides immitis. ${ }^{2}$

Populations at risk for this fungal infection include immunosuppressed patients (HIV, post transplant, etc.), young children, and pregnant women. Additionally, Filipinos, African Americans, Native Americans, Hispanics, and Asians are at greater risk for disseminated coccidioidal disease. ${ }^{1,4,5}$ 
Coccidioidal infections are categorized as primary (pulmonary) or secondary (disseminated). Studies show that of the individuals with primary coccidioidal infections, approximately sixty percent are asymptomatic. The remaining forty percent may present with flu-like symptoms, cough, fever, night sweats, and pleuritic chest pain. Most of these symptoms resolve within 2-3 weeks. Up to one percent of these individuals may go on to develop secondary or disseminated coccidioidal infections. ${ }^{2,4,5,6}$

Disseminated infections may affect the skin, soft tissue structures, bone, joints, and meninges. Skin lesions include papules, abscesses, or pustules. ${ }^{1}$ Synovial lining and joint infections may lead to arthralgia and peri-articular edema, with coccidioidal osteomyelitis occurring in up to fifty percent of patients with disseminated disease. ${ }^{7,8,9}$ Coccidioidal meningitis is the most lethal form of disseminated disease with these infected individuals presenting with nausea, vomiting, headaches, and altered mental status.

The treatment of coccidioidal infections is dependent on the type and severity. In primary pulmonary infections, antifungal therapy is not always recommended as most cases are selflimiting. Treatment is recommended if the patient demonstrates high antibody titers, extensive pneumonia, extrapulmonary disease or is immunosuppressed. Specific therapy includes amphotericin B $0.5-0.7 \mathrm{mg} / \mathrm{kg} /$ day intravenously, ketoconazole $400 \mathrm{mg} /$ day orally, fluconazole 400 $800 \mathrm{mg} /$ day orally or intravenously, or itraconazole $200 \mathrm{mg}$ orally twice daily. ${ }^{10}$ Kushwaha in a 1996 study of 25 cases of coccidioidal osteomyelitis demonstrated that surgical debridement is an indicated component of therapy. ${ }^{11}$ In 1986, Bried and Galgiani completed a study of 24 patients with coccidioidal osteomyelitis and their outcomes also revealed that treatment should include both medical therapy and surgical debridement. ${ }^{5}$

We herein report on a patient with previous primary coccidioidal infection that subsequently progressed to disseminated disease involving the foot and ankle.

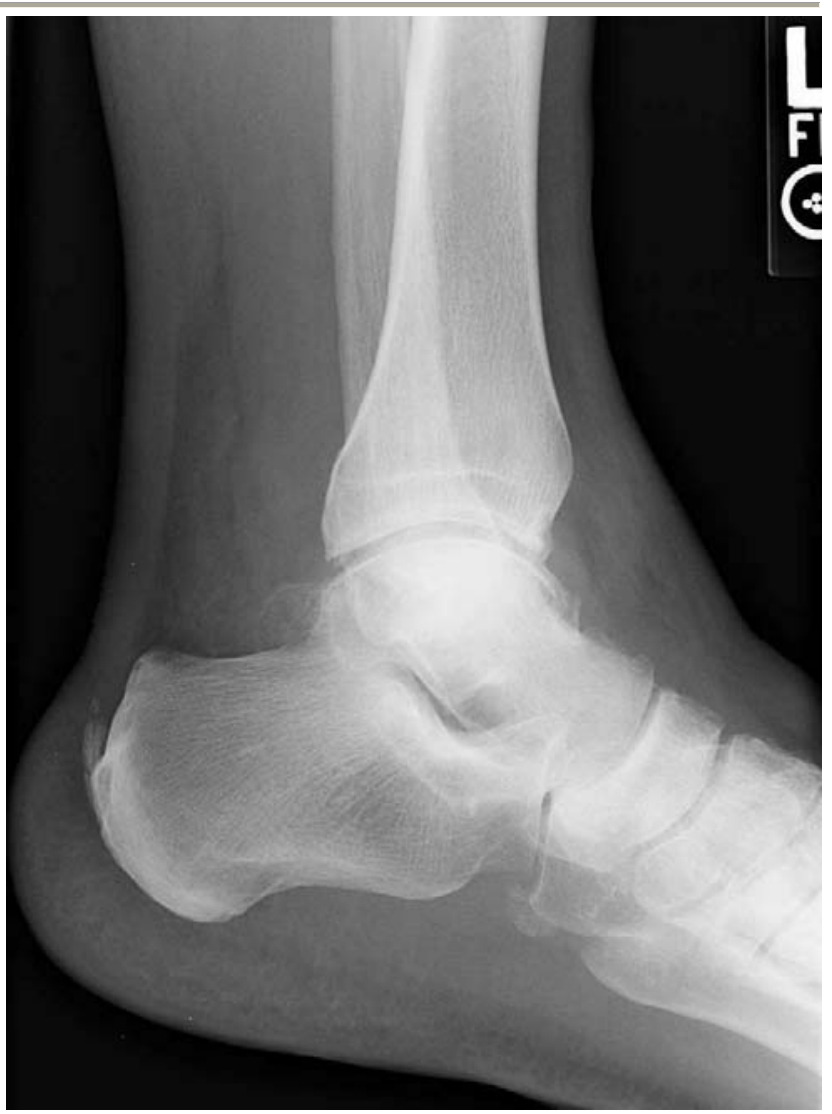

Figure 1 Initial radiographs revealed mild degenerative changes within the left ankle joint, but no other pathology.

\section{Case Report}

A 56 year old male presented to the podiatry department at the Carl T. Hayden VA Medical Center with left ankle pain and swelling of several months duration. After two prior emergency room visits, the patient had only been treated with non steroidal anti-inflammatory agents with no improvement of his pain. During his podiatry clinic visit, the ankle was mildly edematous, had mild pain with range of motion, but no signs of erythema or infection were present. Radiographs revealed mild degenerative changes within the left ankle joint, but no other pathology. (Fig.1) Pertinent past medical history included osteoarthritis, gout, coccidioidal pulmonary infection, hypertension, diabetes mellitus, and renal insufficiency. Ankle joint aspiration was attempted, but did not yield any fluid. The patient was given an intra-articular injection of corticosteroid mixed with a local anesthetic. 


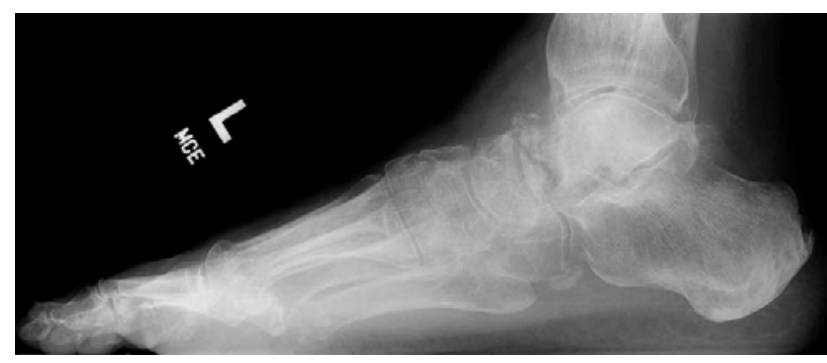

Figure 2 Radiograph of affected foot showing talar neck fracture and diffuse osteopenia subsequently confirmed to be coccidioidomycosis infection.

The patient was seen one week later stating that the injection aggravated his ankle. He was placed in a walking boot and scheduled for a left ankle arthroscopy. Intra-operatively, capsular adhesions with extensive synovitis were noted. In addition, anterior talar dome abnormalities including discoloration and soft cartilage were evident. The remainder of the ankle joint cartilage appeared normal. Post operatively, the patient reported improvement in pain and worked with physical therapy weekly to improve ankle range of motion and strength.

The patient returned to the podiatry clinic three months post ankle arthroscopy with severe ankle pain causing inability to perform daily activities. He had been self treating with non steroidal antiinflammatory agents and an ankle brace with very little relief. On physical exam, the left ankle was noted to be edematous with no evidence of erythema. Pain within the ankle joint and subtalar joint was present with range of motion and palpation. Radiographic findings included osteopenic bone within the rearfoot and a complete fracture of the talar head and neck. Fluffy periostitis was present dorsally and the posterior talus showed periostitis as well. (Fig. 2)

Laboratory testing revealed a normal white cell count with mild elevation of glucose, creatinine, and phosphate. Uric acid was elevated to 9.0, Creactive protein was 2.7 , and the erythrocyte sedimentation rate was high at $65 \mathrm{~mm}$.

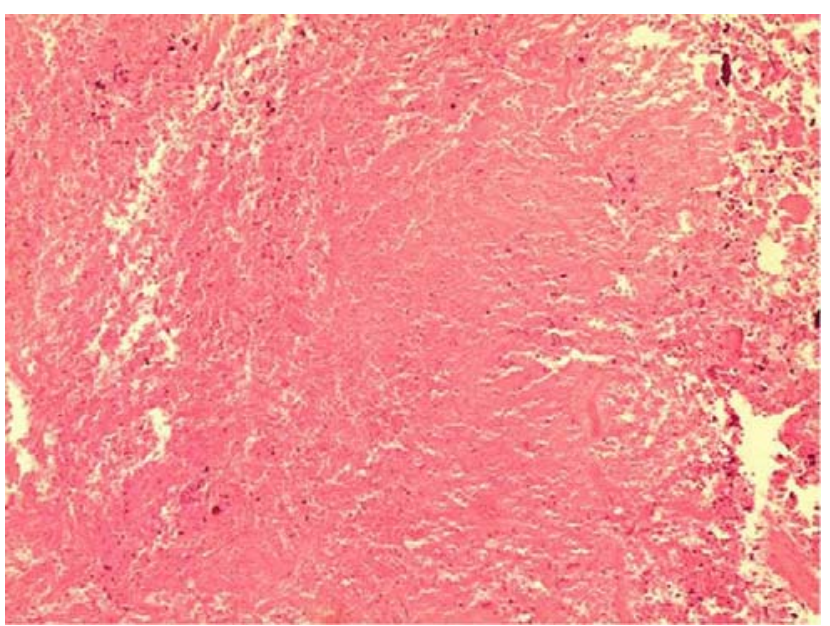

Figure 3 Histopathology showing extensive necrosis of bone and soft tissue with coccidioidal granuloma present within both the talus and the surrounding synovium.

The coccidioides screen was positive and the antibody titer was elevated. Differential diagnoses at this time included; septic arthritis, coccidioides osteomyelitis, gout, osteomyelitis, neoplasm, Charcot arthropathy, and trauma.

A bone biopsy of the talus was then performed and bone cultures evaluated for aerobic, anaerobic, and fungal growth. The pathology report identified extensive necrosis of bone and soft tissue with coccidioidal granuloma present within both the talus and the surrounding synovium. (Fig. 3) No other organisms were identified.

Post operatively the patient was immobilized for 6-8 weeks to allow consolidation of the talar fractures. The patient underwent a full body three phase bone scan to evaluate for any other disseminated locations. Bone activity was noted in several ribs and the left navicular and calcaneus The patient was placed on fluconazole $200 \mathrm{mg}$ twice daily for life by the infectious disease department. Although complete resection of the infected bone was recommended, the patient was not willing to undergo an extensive debridement of the rearfoot. 
At nine months follow up, the patient was ambulating comfortably in regular shoes with an ankle foot orthosis and was continuing with antifungal therapy. He was subsequently lost to follow up.

\section{Discussion}

Although disseminated Coccidioides immitis is rarely a causative organism in osteomyelitis, it should still be included in the list of differential diagnoses when the affected individual resides in an endemic area or has traveled to an endemic area. ${ }^{9}$ Acquisition of the infection occurs with inhalation of the fungal arthrospores which leads to a primary pulmonary infection. This infection is typically self limiting, but can become disseminated to extra-pulmonary sites. ${ }^{8} \quad$ When disseminated to bone, radiographic findings may reveal lytic lesions with periosteal reaction similar to malignancy or bacterial osteomyelitis. ${ }^{8,11}$ Bone biopsy to confirm the diagnosis is recommended. ${ }^{9,11}$ However, joint aspiration may yield fluid for microscopic identification of the coccidioidal spherules in a hematoxylin and eosin stained specimen. Studies indicate that extensive surgical debridement in conjunction with antifungal therapy should be initiated in individuals with coccidioidal osteomyelitis. ${ }^{11}$ Three phase bone scanning may be useful in identifying any further bony involvement. ${ }^{9,11}$

In this case, the patient's initial history included disclosure of previous pulmonary coccidioidomycosis. However, he also had a history of diabetes mellitus, osteoarthritis, and gout. All of these comorbidities could be etiologies of the patient's presenting complaint. Laboratory values indicated no elevation in white blood cell count, but he did have an elevated uric acid. On initial exam, joint aspiration was attempted with no fluid obtained; therefore, no microscopic evaluation of the joint fluid could be performed. After arthroscopy of the ankle, his symptoms resolved.
At his 3 month post operative appointment, follow up radiographs revealed extensive destruction of the talus and subtalar joint area not previously noted on radiographs. After bone biopsy and culture was performed, coccidioidomycosis was the definitive diagnosis.

Due to the extensive infection throughout the entire talus and portions of the calcaneus and navicular, the patient would have likely undergone a proximal amputation due to the need for debridement and resection of such a large amount of bone. The patient and the podiatry team agreed to attempt suppression of the infection with fluconazole, serial radiographs, and periodic follow up visits. Unfortunately, he left our care nine months later.

In conclusion, disseminated coccidioidomycosis is an aggressive and destructive form of osteomyelitis that requires lifelong therapy. Although the infection is rare, coccidioidomycosis should be considered in individuals living in the endemic areas of the southwestern United States. Early recognition and treatment are important to prevent loss of function and extensive surgical debridement of the affected limb. 


\section{References}

1. Galgiani, J: Coccidioidomycosis. The Western Journal of Medicine 159 (2): 153 - 181, August 1993.

2. Stevens, D: Coccidioidomycosis. The New England Journal of Medicine 332 (16): 1077-1082, April 1995.

3. Panackal A, Haffeh R, Cetron M, et al: Fungal Infections Among Returning Travelers. Clinical Infectious Disease 35:1088-1095, 2002.

4. Drutz: Coccidioidomycosis Part 1. American Rev Respiratory Diseases 117: 559-585, 1978.

5. Bried J, Galgiani J: Coccidioides immitis Infections in Bones and Joints. Clinical Orthopedics and Related Research 211:235-243, October 1986.

6. May D, Disler D: Case 50 Primary Coccidioidomycosis of the Knee. Radiology

September 224: 3, 2002.

7. Sprinkle R, Kosova L, Tougas T, et al: Disseminated Coccidioidomycosis of an Ankle Joint. Journal of the Podiatric Medical Association 79 (6): 300-305, 1989.

8. Buckley, Steven: Coccidioidomycosis of the First Cuneiform: Successful

Treatment Using Local Debridement and Long Term Ketoconazole Therapy.

Foot and Ankle 6: June 1986

9. Sandoval J, Shank J, Morgan S, et al: Midfoot Coccidioidal Osteomyelitis. A Case Report and Review of the Literature. Journal of Bone and Joint Surgery Am. 88: 861-865, 2006.

10. Galgiani J, Ampel N, Catanzaro A, et al: Practice Guidelines for the Treatment of Coccidioidomycosis. Clinical Infectious Disease 30: 658-661, 2000.

11. Kushwaha V, Shaw B, Gerardi J, et al: Musculoskeletal Coccidioidomycosis. A Review of 25 Cases. Clinical Orthopedics and Related Research 332: 190-199, November 1996. 